\title{
ON THE LIMIT RELATION FOR THE QUANTUM RELATIVE ENTROPY
}

\author{
J. Z. BERNÁD \\ Institut für Angewandte Physik, Technische Universität Darmstadt, \\ D-64289 Darmstadt, Germany
}

A. B. FRIGYIK

Institute of Mathematics and Informatics, University of Pécs, H-7624

Pécs, Hungary

\begin{abstract}
Recently, Diósi et al. 1 introduced a simple, yet very interesting model for reservoirs, in order to study the relationship between thermodynamic entropy production of a system and the corresponding von Neumann entropy (or informatic entropy, as it was called by the authors). They came up with a conjecture about the asymptotic behaviour of the state introduced in the model. The conjecture was proven later by Csiszár et al. 2]. In this paper we give a different interpretation of the original question and extend the result to a collection of more general states.
\end{abstract}

\section{INTRODUCTION}

In a thermodynamic model, Diósi et al. [1] considered an elementary model of a reservoir, in which the thermodynamic entropy production due to the application of an external field equals the change of the informatic entropy. Let us recapitulate the main ideas of this model. It has been assumed that this reservoir is composed by $n$ distinguishable particles with finite energy levels. The time evolution of these particles is governed by the non-interacting Hamilton operator

$$
H_{R}=H \otimes I^{\otimes(n-1)}+I \otimes H \otimes I^{\otimes(n-2)}+\cdots+I^{\otimes(n-1)} \otimes H .
$$

E-mail addresses: zsolt.bernad@physik.tu-darmstadt.de, afrigyik@gamma.ttk.pte.hu.

Date: September 25, 2018. 
Each particle is considered to be in a Gibbs state with a fixed inverse temperature $\beta$ and the state of the reservoir is assumed to be completely uncorrelated:

$$
\rho_{R}=\rho^{\otimes n}, \quad \rho=\frac{\mathrm{e}^{-\beta H}}{\operatorname{Tr}\left\{\mathrm{e}^{-\beta H}\right\}} .
$$

An external field is applied instantaneously on the reservoir, i.e a unitary evolution takes place on a much smaller time scale than the time scale of the reservoir's evolution, and it is assumed that only one of the particles changes its state to $\sigma$ :

$$
\sigma=U \rho U^{*}, \quad[U, H] \neq 0,
$$

where $U$ is a unitary operator. This results in $n$ possible reservoir states $\rho_{R}^{\prime}$

$$
\sigma \otimes \rho^{\otimes(n-1)}, \rho \otimes \sigma \otimes \rho^{\otimes(n-2)}, \ldots, \rho^{\otimes(n-1)} \otimes \sigma,
$$

depending which particle changed its state. The reservoir changes its mean energy by

$$
\Delta E=\operatorname{Tr}\left\{H_{R} \rho_{R}^{\prime}\right\}-\operatorname{Tr}\left\{H_{R} \rho_{R}\right\}=\beta^{-1} S(\sigma \| \rho)>0
$$

where $S(\sigma \| \rho)$ is the relative entropy and we used the relation $\log \rho=-\beta H-$ $\log \left(\operatorname{Tr}\left\{\mathrm{e}^{-\beta H}\right\}\right)($ see Eq. (1) ) together with the fact that the von Neumann entropy is unitarily invariant:

$$
S(\sigma)=-\operatorname{Tr}\{\sigma \log (\sigma)\}=-\operatorname{Tr}\left\{U \rho U^{*} \log \left(U \rho U^{*}\right)\right\}=S(\rho) .
$$

If this mean energy is dissipated in the reservoir, then the thermodynamic entropy production is equal to $S(\sigma \| \rho)$. But the informatic entropy production is zero:

$$
S\left(\rho_{R}^{\prime}\right)-S\left(\rho_{R}\right)=0 .
$$

The authors of Ref. [1] needed a new state $R_{n}$ instead of $\rho_{R}^{\prime}$ such that

$$
\operatorname{Tr}\left\{H_{R} \rho_{R}^{\prime}\right\}=\operatorname{Tr}\left\{H_{R} R_{n}\right\} \quad \text { and } \quad S\left(R_{n}\right)-S\left(\rho_{R}^{\prime}\right) \rightarrow S(\sigma \| \rho)
$$

in the thermodynamic limit $n \rightarrow \infty$. It was conjectured that for

$$
R_{n}=\frac{\sigma \otimes \rho^{\otimes(n-1)}+\rho \otimes \sigma \otimes \rho^{\otimes(n-2)}+\cdots+\rho^{\otimes(n-1)} \otimes \sigma}{n}
$$

the informatic entropy production rate is equal to the relative entropy $S(\sigma \| \rho)$ in the thermodynamic limit. This conjecture was proved by Csiszár et al. [2]. The proof generalizes the conjecture of Ref. [1] for arbitrary density matrices $\sigma$ and $\rho$, which are not related any more by a unitary transformation, i.e. possibly $S\left(\rho_{R}^{\prime}\right) \neq S\left(\rho_{R}\right)$, and shows that

$$
\begin{aligned}
& \lim _{n \rightarrow \infty}\left(S\left(R_{n}\right)-S\left(\rho_{R}^{\prime}\right)\right)=S(\sigma \| \rho), \\
& \forall \rho_{R}^{\prime} \in\left\{\sigma \otimes \rho^{\otimes(n-1)}, \rho \otimes \sigma \otimes \rho^{\otimes(n-2)}, \ldots, \rho^{\otimes(n-1)} \otimes \sigma\right\} .
\end{aligned}
$$

This whole problem can be viewed from a different angle. Let us consider the approach of M. J. Donald [3] and calculate the Helmholtz free energy $F$ 
of the reservoir in the state $R_{n}$ and $\rho_{R}=\rho^{\otimes n}$. The difference between them is given by

$$
F\left(R_{n}\right)-F\left(\rho^{\otimes n}\right)=\beta^{-1} S\left(R_{n} \| \rho^{\otimes n}\right) .
$$

One can simply compute

$$
S\left(R_{n} \| \rho^{\otimes n}\right)=-S\left(R_{n}\right)+S\left(\rho_{R}^{\prime}\right)+S(\sigma \| \rho) .
$$

Thus Eq. (22) is equivalent to

$$
S\left(R_{n} \| \rho^{\otimes n}\right) \rightarrow 0 \quad \text { as } \quad n \rightarrow \infty
$$

which yields

$$
F\left(R_{n}\right)-F\left(\rho^{\otimes n}\right) \rightarrow 0 \quad \text { as } \quad n \rightarrow \infty .
$$

This means that in the thermodynamic limit the reservoir changed its equilibrium state to another equilibrium state. A natural question is to find all these thermodynamically equivalent states. In case when only one particle changed its Gibbs state $\rho$ to an arbitrary state $\sigma$, the proof in Ref. 2] shows that the state $R_{n}$ - where the randomization is uniform, i.e. each individual state has the same probability to change to state $\sigma$ - is an equilibrium state equivalent to the inital state $\rho^{\otimes n}$.

In this paper we consider a general case: We suppose that the randomization is not uniform, hence we consider the state

$$
\rho_{n}=a_{n, 1} \sigma \otimes \rho^{\otimes(n-1)}+a_{n, 2} \rho \otimes \sigma \otimes \rho^{\otimes(n-2)}+\cdots+a_{n, n} \rho^{\otimes(n-1)} \otimes \sigma,
$$

where the matrix $\left(a_{i, j}\right)$ contains the changing weights for all $n$, such that $a_{n, i} \in[0,1]$ when $i \leqslant n$ otherwise $a_{n, i}=0$ and $\sum_{i=1}^{n} a_{n, i}=1$. This construction allows us to study the thermodynamic limit $n \rightarrow \infty$. The main question is about the properties of this matrix that assure the following limit:

$$
S\left(\rho_{n} \| \rho^{\otimes n}\right) \rightarrow 0 \quad \text { as } \quad n \rightarrow \infty .
$$

In general, a matrix $\left(a_{i, j}\right)$ is regular or T-matrix (see Ref. [4]), if the following conditions are satisfied:

$$
\begin{aligned}
& \sup _{n \in \mathbb{N}^{+}} \sum_{i=1}^{n}\left|a_{n, i}\right|<\infty, \quad \lim _{n \rightarrow \infty} \sum_{i=1}^{n} a_{n, i}=1, \\
& \lim _{n \rightarrow \infty} a_{n, j}=0, \quad \forall j \in \mathbb{N}^{+} .
\end{aligned}
$$

It is strongly regular if it is regular and (see Ref. [5])

$$
\lim _{n \rightarrow \infty} \sum_{j=1}^{\infty}\left|a_{n, j+1}-a_{n, j}\right|=0 .
$$

We are going to give an analytical proof of the following statement:

Theorem 1.1. Let $\rho$ be a Gibbs state and $\sigma$ a state such that $\operatorname{supp}(\sigma) \subseteq$ $\operatorname{supp}(\rho)$. Then for every strongly regular matrix $\left(a_{i, j}\right)$, where $a_{i, j} \geq 0$ for all $i, j \in \mathbb{N}^{+}$and $\sum_{i=1}^{n} a_{n, i}=1$ for all $n \in \mathbb{N}^{+}$, and

$$
\rho_{n}=a_{n, 1} \sigma \otimes \rho^{\otimes(n-1)}+a_{n, 2} \rho \otimes \sigma \otimes \rho^{\otimes(n-2)}+\cdots+a_{n, n} \rho^{\otimes(n-1)} \otimes \sigma
$$


we have

$$
\lim _{n \rightarrow \infty} S\left(\rho_{n} \| \rho^{\otimes n}\right)=0 .
$$

The entries of the strongly regular matrix $\left(a_{i, j}\right)$ must be restricted to the interval $[0,1]$ in order for the state in (11) to be a real physical state of the reservoir. Thus, Theorem 1.1 is capable to identify a large class of states for which the Helmholtz free energy in the thermodynamic limit is the same as for the equilibrium state $\rho^{\otimes n}$, where $n \in \mathbb{N}^{+}$. The presented proof combines the idea that comes from Ref. [2] with results from ergodic theory [6].

\section{GNS APPROACH}

Let us start with Eq. (11): Applying the same idea that was used in Ref. 2 we can rewrite $\rho_{n}$ in a more suitable form. Let $X=\rho^{-1 / 2} \sigma \rho^{-1 / 2}$, where $\rho$ is invertible (see definition in (11)). Then we have

$\rho_{n}=\left(\rho^{\frac{1}{2}}\right)^{\otimes n}\left(a_{n, 1} X \otimes I^{\otimes(n-1)}+a_{n, 2} I \otimes X \otimes I^{\otimes(n-2)}+\cdots+a_{n, n} I^{\otimes(n-1)} \otimes X\right)\left(\rho^{\frac{1}{2}}\right)^{\otimes n}$.

Here, we cannot use directly the mean ergodic theorem that was applied in Ref. 2] or Ref. [7] (see Proposition 1.17 there). Nevertheless, we will follow a very similar path to that which was given in the proof of the aforementioned proposition. The algebra underlying our problem is the same as the proposition's and hence we are going to adopt the notation used in Ref. [7. As we have already stated, the reservoir consists of particles with finite energy levels, and we denote by $d$ the dimension of the Hilbert space underlying their quantum mechanical description. Let $M_{d}$ denote the set of all $d \times d$ complex matrices acting on this Hilbert space. Since $M_{d}$ is a $C^{*}$-algebra with identity, we consider the family of $C^{*}$-algebras $\left\{M_{d^{n}}=\left(M_{d}\right)^{\otimes n} \mid n \in \mathbb{N}^{+}\right\}$the $n$-fold algebraic tensor products of $M_{d}$. If we identify $M_{d^{n}}$ with $M_{d^{n}} \otimes I$ in $M_{d^{n+1}}$, where $I=I_{d}$ denotes the identity operator on the $d$ dimensional Hilbert space, we can consider $M_{\infty}=\bigcup_{n=1}^{\infty} M_{d^{n}}$, the inductive limit $*$-algebra of $\left\{M_{d^{n}}\right\}$, and its completion $A_{\infty}$. In other words,

$$
A_{\infty}=\overline{\bigotimes_{i=1}^{\infty} A_{i}}
$$

where $A_{i}=M_{d}$ for all $i \in \mathbb{N}^{+}$(for the details see Ref. [8], Section 1.23). The family of faithful states (see Eq. (11) $\left\{\varphi_{n}(x)=\operatorname{Tr}\left\{\rho^{\otimes n} x\right\} \mid x \in M_{d^{n}}, n \in\right.$ $\left.\mathbb{N}^{+}\right\}$, are functionals on the particular $C^{*}$-algebras, which extend to a faithful state $\varphi_{\infty}$ on $A_{\infty}$. The GNS representation of $A_{\infty}$ with respect to the state $\varphi_{\infty}$ results in a triplet $(\pi, \mathcal{H}, \Omega)$, where $\mathcal{H}$ is the constructed Hilbert-space, $\pi$ is a $*$-homomorphism of $A_{\infty}$ into the set of all bounded operators $\mathcal{B}(\mathcal{H})$ on $\mathcal{H}$, and $\Omega$ is the cyclic vector for $\pi$. We generate the von Neumann algebra $\mathcal{M}=\pi\left(A_{\infty}\right)^{\prime \prime}$ by taking the bicommutant of $\pi\left(A_{\infty}\right)$ and thus we make sure that all weakly or strongly convergent series have their limit point in $\mathcal{M}$. 
Just like in Ref. [7] we are going to denote by $\gamma$ the right shift on $A_{\infty}$ which is defined for $x_{1} \otimes x_{2} \otimes \cdots \otimes x_{n} \in M_{d^{n}}$ as

$$
\gamma\left(x_{1} \otimes x_{2} \otimes \cdots \otimes x_{n}\right)=I \otimes x_{1} \otimes x_{2} \otimes \cdots \otimes x_{n} \in M_{d^{n+1}} .
$$

As a result, $\rho_{n}$ can be written as

$$
\rho_{n}=\left(\rho^{1 / 2}\right)^{\otimes n}\left(\sum_{i=0}^{n-1} a_{n, i+1} \gamma^{i}(X)\right)\left(\rho^{1 / 2}\right)^{\otimes n}
$$

It is obvious that $\varphi_{\infty}$ is invariant under $\gamma$. The extension $\varphi$ of the state $\varphi_{\infty}$ to $\mathcal{M}$ is

$$
\varphi(a)=\langle\Omega, a \Omega\rangle, a \in \mathcal{M},
$$

and the right shift $\gamma$ can be also extended such that $\varphi \circ \gamma=\varphi$. Now, the only missing link in this approach is the connection between the relative entropy $S\left(\rho_{n} \| \rho^{\otimes n}\right)$ and the state $\varphi$. Let us recall the Belavkin-Staszewski entropy and its relation to the relative entropy (see Ref. [2] and Proposition 7.11 in Ref. [7])

$$
S\left(\rho_{n} \| \rho^{\otimes n}\right) \leqslant S_{B S}\left(\rho_{n} \| \rho^{\otimes n}\right)=\operatorname{Tr}\left\{\rho^{\otimes n} \eta\left(\left(\rho^{-1 / 2}\right)^{\otimes n} \rho_{n}\left(\rho^{-1 / 2}\right)^{\otimes n}\right)\right\}
$$

where $\eta(t)=-t \log t$ and $\operatorname{supp}(\sigma) \subseteq \operatorname{supp}(\rho)$. One can simply realize that the right hand side of the above equation is nothing else than the state $\varphi_{n}$ and therefore with the help of the GNS construction we get

$$
S\left(\rho_{n} \| \rho^{\otimes n}\right) \leq\left\langle\Omega, \eta\left(\sum_{i=0}^{n-1} a_{n, i+1} \gamma^{i}(\mathcal{X})\right) \Omega\right\rangle,
$$

where $\Omega$ is the cyclic vector and $\mathcal{X} \in \mathcal{M}$ is the embedding of $X=\rho^{-1 / 2} \sigma \rho^{-1 / 2}$ into $\mathcal{M}$.

\section{Proof of Theorem 1.1}

We would like to prove first that $\sum_{i=0}^{n-1} a_{n, i+1} \gamma^{i}(\mathcal{X}) \rightarrow \mathrm{id}_{\mathcal{H}}$ strongly. In order to do that we need the following theorem (Theorem 3.1) from Ref. [5] (see Ref. 9] pg. 213 for the original theorem). For convenience of the reader we repeat a slightly modified version of the statement here.

Theorem 3.1. Let $Y$ be a Banach space and let $T: Y \rightarrow Y$ be a continuous linear operator such that the family of operators $\left\{T^{n}, n \in \mathbb{N}\right\}$ is power bounded. Further, let $A=\left(a_{n, i}\right)\left(n, i \in \mathbb{N}^{+}\right)$be a strongly regular matrix and

$$
T_{n}=\sum_{i=1}^{\infty} a_{n, i} T^{i}, \quad\left(n \in \mathbb{N}^{+}\right) .
$$

Then the strong limit $T_{0}=\lim _{n \rightarrow \infty} T_{n}$ exists and it is a projection on $Y$ onto the subspace $\mathcal{F}=\{y \in Y: T y=y\}$ of all fixed points of $T$. Moreover, projection $T_{0}$ does not depend on the choice of the strongly regular matrix $A$. 
The GNS construction provides us with a Hilbert space $\mathcal{H}$ which is a reflexive Banach space and for the rest of the paper we are going to adopt the proof of Proposition 1.17 in Ref. [7]. Let $V: \mathcal{H} \rightarrow \mathcal{H}$ be defined by

$$
V a \Omega=\gamma(a) \Omega, \quad \forall a \in \mathcal{M} .
$$

The map $V: \mathcal{H} \rightarrow \mathcal{H}$ is an isometry on $\mathcal{A}_{\infty} \Omega$ :

$$
\begin{aligned}
& \langle V a \Omega, V b \Omega\rangle=\langle\gamma(a) \Omega, \gamma(b) \Omega\rangle=\langle(I \otimes a) \Omega,(I \otimes b) \Omega\rangle \\
& =\left\langle\Omega,\left(I \otimes a^{*}\right)(I \otimes b) \Omega\right\rangle=\left\langle\Omega,\left(I \otimes a^{*} b\right) \Omega\right\rangle=\varphi_{\infty}\left(I \otimes a^{*} b\right) \\
& \quad=\varphi_{\infty}(I) \varphi_{\infty}\left(a^{*} b\right)=\varphi_{\infty}\left(a^{*} b\right)=\left\langle\Omega, a^{*} b \Omega\right\rangle=\langle a \Omega, b \Omega\rangle .
\end{aligned}
$$

Here, we used the fact that $\varphi_{\infty} \uparrow_{d^{n}}=\varphi_{n}$. Since $V$ is an isometry and $\overline{\pi\left(\mathcal{A}_{\infty}\right) \Omega}=\mathcal{H},\left\{V^{n}, n \in \mathbb{N}\right\}$ is a power bounded family on $\mathcal{H}$, which means that we can apply Theorem 3.1 to our situation, where $A=\left(a_{i, j}\right)$ is a strongly regular matrix. The isometry $V$ plays the role of $T$, hence we need to find the fixed point subspace of $V$. Since the matrix $A$ does not play any role in finding this subspace, the proof of Proposition 1.17 in Ref. [7] works verbatim and therefore the dimension of $\mathcal{F}$ is one. Since

$$
V \operatorname{id}_{\mathcal{A}_{\infty}} \Omega=\gamma\left(\operatorname{id}_{\mathcal{A}_{\infty}}\right) \Omega=\gamma\left(I^{\otimes \infty}\right) \Omega=I^{\otimes \infty} \Omega=\operatorname{id}_{\mathcal{A}_{\infty}} \Omega,
$$

the $\Omega$ is in $\mathcal{F}$ and hence $\mathcal{F}=\mathbb{C} \Omega$.

If $b^{\prime} \in \mathcal{M}^{\prime}$, where $\mathcal{M}^{\prime}$ is the commutant of $\mathcal{M}$, and $S_{n}(a)=\sum_{i=0}^{n-1} a_{n, i+1} \gamma^{i}(a)$, $a \in \mathcal{M}$, then

$$
S_{n}(a) b^{\prime} \Omega=b^{\prime} S_{n}(a) \Omega=b^{\prime} \sum_{i=0}^{n-1} a_{n, i+1} \gamma^{i}(a) \Omega=b^{\prime} \sum_{i=0}^{n-1} a_{n, i+1} V^{i} a \Omega \rightarrow b^{\prime} E a \Omega,
$$

by Theorem 3.1, where $E$ is the projection of $\mathcal{H}$ onto $\mathcal{F}: E(\xi)=\langle\Omega, \xi\rangle \Omega$ for all $\xi \in \mathcal{H}$. Thus

$$
b^{\prime} E a \Omega=b^{\prime}\langle\Omega, a \Omega\rangle \Omega=\varphi(a) b^{\prime} \Omega .
$$

We know from the proof of Proposition 1.17 in Ref. [7] that $\Omega$ is cyclic for $\mathcal{M}^{\prime}$, as well, hence $S_{n}(a) \rightarrow \varphi(a)$ id $_{\mathcal{H}}$ strongly. If we choose $a=\mathcal{X}$ then

$$
\varphi(\mathcal{X})=\operatorname{Tr}\left\{\rho \rho^{-1 / 2} \sigma \rho^{-1 / 2}\right\}=1 .
$$

Hence $S_{n}(\mathcal{X}) \rightarrow \operatorname{id}_{\mathcal{H}}$ strongly.

We can finish the proof now. Operator $\mathcal{X}$ is positive and the function $\eta(t)=-t \log t$ is continuous on $[0, \infty)$. Since the continuous functional calculus preserves the strong convergence, we obtain that $\lim _{n \rightarrow \infty} \eta\left(S_{n}(\mathcal{X})\right)=0$ strongly. Therefore, with the aid of Eq. (3)

$$
\lim _{n \rightarrow \infty}\left\langle\Omega, \eta\left(\sum_{i=0}^{n-1} a_{n, i+1} \gamma^{i}(\mathcal{X})\right) \Omega\right\rangle=0 \Rightarrow \lim _{n \rightarrow \infty} S\left(\rho_{n} \| \rho^{\otimes n}\right)=0
$$

and the proof is complete.

Similarly, one can also obtain for

$$
\rho_{n, k}=a_{n, 1} \sigma^{\otimes k} \otimes \rho^{\otimes(n-k)}+a_{n, 2} \rho \otimes \sigma^{\otimes k} \otimes \rho^{\otimes(n-k-1)}+\cdots+a_{n, n-k+1} \rho^{\otimes(n-k)} \otimes \sigma^{\otimes k}
$$


with $\mathcal{X}=\rho^{-1 / 2} \sigma^{\otimes k} \rho^{-1 / 2}, \sum_{i=1}^{n-k+1} a_{n, i}=1$ and fixed $k$ the limit relation $\lim _{n \rightarrow \infty} S\left(\rho_{n, k} \| \rho^{\otimes n}\right)=0$.

This result extends a similar statement made in Ref. [2] and further extends the set of states identified in Theorem 1.1.

\section{ACKNOWLEDGEMENT}

This work was supported by the Deutscher Akademischer Austauschdienst (MÖB-DAAD project no. 65049).

\section{REFERENCES}

[1] L. Diósi, T. Feldmann, and R. Kosloff, Int. J. Quant. Inf. 4, 99 (2006).

[2] I. Csiszár, F. Hiai, and D. Petz, J. Math. Phys. 48, 092102 (2007).

[3] M. J. Donald, J. Stat. Phys. 49, 81 (1987).

[4] R. G. Cooke, Infinite Matrices and Sequence Spaces (MacMillan, London, 1950).

[5] C. Jardas and N. Sarapa, Math. Commun. 2, 107 (1997).

[6] U. Krengel, Ergodic Theorems (de Gruyter, Berlin, 1985).

[7] M. Ohya and D. Petz, Quantum Entropy and its Use (Springer-Verlag, New York, 1993).

[8] S. Sakai, $C^{*}$-Algebras and $W^{*}$-Algebras (Springer-Verlag, Berlin, 1971).

[9] K. Yosida, Functional analysis. Classics in Mathematics. Reprint of the 1980 Edition. (Springer-Verlag, Berlin Heidelberg, 1995). 